\title{
The mm-to-cm SED of spiral galaxies
}

\section{Synergies between NIKA2 and SRT instruments}

\author{
Simone Bianchi ${ }^{1, *}$, Matteo Murgia ${ }^{2}$, Andrea Melis $^{2}$, Viviana Casasola ${ }^{3}$, Maud Galametz ${ }^{4}$, \\ Frédéric Galliano ${ }^{4}$, Federica Govoni ${ }^{2}$, Anthony Jones ${ }^{5}$, Suzanne Madden ${ }^{4}$, Rosita Paladino ${ }^{3}$, \\ Emmanuel Xilouris ${ }^{6}$, and Nathalie Ysard $^{5}$ \\ ${ }^{1}$ INAF-Osservatorio Astrofisico di Arcetri, L. E. Fermi 50, 50125, Firenze, Italy \\ ${ }^{2}$ INAF-Osservatorio Astronomico di Cagliari, Via della Scienza 5, 09047 Selargius (CA), Italy \\ ${ }^{3}$ INAF-Istituto di Radioastronomia-Via P. Gobetti, 101, 40129 Bologna, Italy \\ ${ }^{4}$ AIM, CEA, CNRS, Université Paris-Saclay, Université Paris Diderot, Sorbonne Paris Cité, 91191 Gif- \\ sur-Yvette, France \\ ${ }^{5}$ Université Paris-Saclay, CNRS, Institut d'Astrophysique Spatiale, 91405 Orsay, France \\ ${ }^{6}$ National Observatory of Athens, Institute for Astronomy, Astrophysics, Space Applications and Re- \\ mote Sensing, Ioannou Metaxa and Vasileos Pavlou, 15236 Athens, Greece
}

\begin{abstract}
The mm-to-cm range of the Spectral Energy Distribution of spiral galaxies remains largely unexplored. Its coverage is required to disentangle the contribution of dust emission, free-free and synchrotron radiation and can provide constraints on dust models, star-formation rates and ISM properties. We present the case for a synergy between NIKA2 observations of nearby spirals and those from planned and current instrumentation at the Sardinia Radio Telescope, and report on a pilot K-band program to search for Anomalous Microwave Emission, an elusive emission component which is presumably related to dust.
\end{abstract}

\section{Galactic SEDs in the mm-to-cm range}

Our knowledge of the Spectral Energy Distribution (SED) of nearby galaxies has greatly improved in recent times, thanks to multi-wavelength surveys and in particular to the Herschel Space Observatory. Herschel has extended the SED coverage to far-infrared (FIR) and submm wavelengths, a range where dust, i.e. solid grains in the interstellar medium (ISM), emits the radiation it has absorbed from starlight $(\sim 1 / 4$ of the total luminosity for late type galaxies; [1]). Using a dust model and fitting tools such as, e.g., CIGALE [2] and HerBIE [3], a wealth of information can be extracted from the SED: star-formation rates (SFRs), stellar and dust masses, gas-to-dust ratios, etc. ([4-7], just to mention a few recents works). Figure 1 shows an example of the current SED knowledge: it uses data from DustPedia [8], a database collecting photometry and imagery for all the large $\left(D_{25}>1^{\prime}\right)$ and nearby $(d<30 \mathrm{Mpc})$ galaxies observed by Herschel; and mid radio continuum flux densities from [9].

A few gaps still remain: in particular, data for $15 \leq v / \mathrm{GHz} \leq 300$ (mm-to-cm range) is limited to the Milky Way (MW) and a few bright objects. The high-frequency side of this gap, at $v \approx 200 \mathrm{GHz}$, is still dominated by dust emission: radiation could come from

\footnotetext{
*e-mail: simone.bianchi@inaf.it
} 


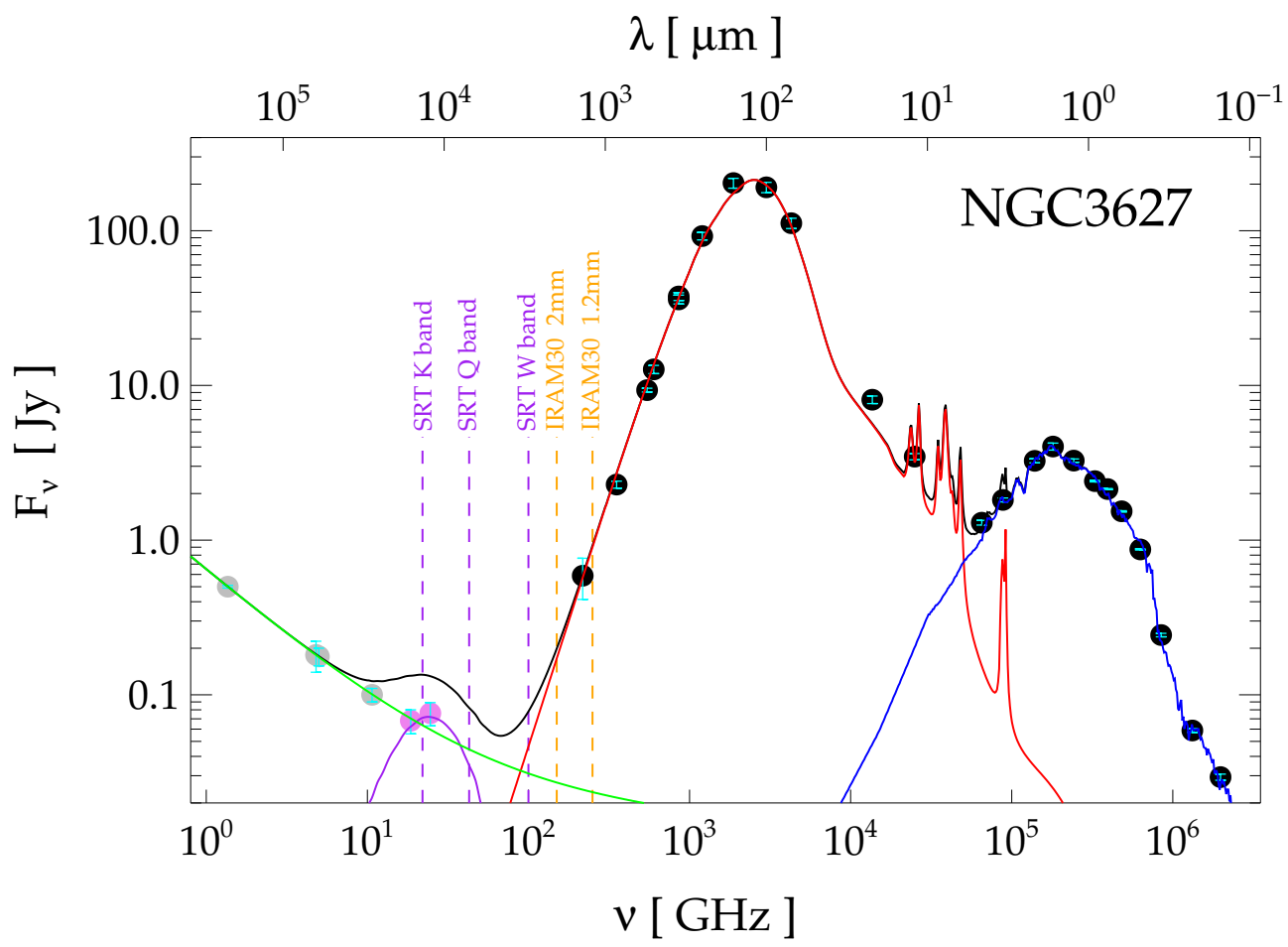

Figure 1. The SED of NGC3627. The total SED (black) results from the sum of: 1) CIGALE fits of the stellar (blue line) and dust (red line) components to the DustPedia datapoints (black dots; [4, 10]); 2) synchrotron and free-free radiation fits to the mid-radio continuum emission (green line and grey dots; [9]); 3) a prediction for AME (purple line), obtained using a model spectral template [11] normalised to the MW AME(30 GHz)/IR(100 $\mu \mathrm{m})$ ratio [12]. NIKA2 and the SRT instrumentation can cover the $\mathrm{mm}$-to-cm gap. DustPedia datapoints at $\mathrm{mm}$ wavelengths are from (unresolved) Planck observations, while the purple datapoints show the results from our pilot SRT program in the K band. DustPedia images and photometry are available at http://dustpedia.astro.noa.gr/.

the Rayleigh-Jeans tail of the warmer dust, or be due to colder grains undetected from FIRsubmm observations. Large masses of cold grains, and/or variations of the dust absorption cross-section at longer wavelengths could be responsible for the submm excess detected in a few objects [13]. Thus, observations at these frequencies are important to constrain the properties of dust, which are still uncertain. Current dust models are made to reproduce the observations of extinction, emission and depletion at high galactic latitude in the MW [14]. Various dust models exist, leading to different estimates of quantities such as the dust mass (see, e.g., [4]). Furthermore, recent lab measurements are questioning the validity of the optical properties generally assumed for the grain materials [15]. Finally, there are indications that the bulk of dust in a galaxy might have different properties than those of the diffuse MW medium $[16,17]$. The NIKA2 camera at the IRAM 30m radiotelescope, and in particular the IMEGIN guaranteed-time project observing large nearby galaxies at millimetre wavelengths $[18,19]$, will help in shedding light on these issues.

At lower frequencies, emission components other than dust need to be taken into account. Already in NIKA2 maps at $2 \mathrm{~mm}$, about $10 \%$ of the SED is due to free-free and synchrotron 
radiation; their contribution increases, and that of dust reduces, in the mid and low-frequency side of the $15 \leq v / \mathrm{GHz} \leq 300$ gap (see, e.g.. [18]). Free-free emission gives the maximum contribution in the mid of the frequency gap. Being due mostly to free electrons, this radiation can be directly related to ionising photons and thus provides a direct estimate of the starformation rate, unaffected by the dust extinction corrections needed for estimates based on the UV continuum and the hydrogen recombination lines in the optical [20]; or by the uncertain fraction of dust heating due to young stars, for estimates based on infrared dust emission [4]. The dominant emission toward the low-frequency side of the gap is due to synchrotron radiation, produced by cosmic ray electrons accelerated to relativistic velocities in supernova remnants and spiralling along the magnetic field in the ISM [20]. Since all of these emission mechanisms overlap in frequency, full SED coverage is needed to solve the degeneracies and estimate the exact contribution of each component.

An opportunity to extend the knowledge of the SED beyond the frequencies accessible to the NIKA2 camera is soon going to be offered at the Sardinia Radio telescope (SRT [21]; see Fig. 1). In the incoming year, the MISTRAL camera, operating in the $\mathrm{W}$ Band at $\sim 90 \mathrm{GHz}$, will be installed at SRT [22], as well as a 19-feed Q band receiver at $\sim 40 \mathrm{GHz}$ [23]. Thanks to the large SRT antenna, $64 \mathrm{~m}$, these instruments will allow us to map nearby galaxies with $\mathrm{HPBW}=12^{\prime \prime}$ and $28^{\prime \prime}$, respectively, resolutions close to those of NIKA2 and the Herschel instruments. Already available, a 7-feed spectro-polarimetric receiver allows access to the $\mathrm{K}$ band at $\sim 22 \mathrm{GHz}[24]$.

\section{Anomalous Microwave Emission}

A further emission component might need to be considered: the Anomalous Microwave Emission (AME). First detected in the MW in the mid-90s by Cosmic Microwave Background experiments, AME is seen as an excess over the expected free-free and synchrotron emissions, peaking at $\approx 30 \mathrm{GHz}$ and correlating with dust emission at larger frequencies (see [25] for a review). AME is seen both in isolated clouds [26], as well as in the diffuse MW medium where its amplitude, relative to the $100 \mu \mathrm{m}$ surface brightness, is $\operatorname{AME}(30 \mathrm{GHz}) / \mathrm{I}(100 \mu \mathrm{m}) \approx 1 / 3000$ [12].

The most accredited theory explains AME with electric dipole emission from rapidly rotating grains [27]. For typical ISM conditions, these grains must have size of $10^{-9} \mathrm{~m}$, in order to spin fast and emit at the AME frequencies: they could be associated with the macromolecules carriers of the PAH features in the mid-infrared spectrum [28], or with nanosilicates [29]. Alternatively, AME could be due to an enhancement of the absorption/emission cross sections at microwave frequencies, either due to magnetic inclusions in grains [30] or the amorphous state of the grain material [31,32]. AME can thus set constraints on the grain size distribution (and ISM conditions), under the spinning grain hypothesis, and/or on the grain composition, for the thermal emission hypothesis. Though these models can all explain AME, yet there is no concluding evidence for its physical mechanism: some authors find a good correlation with small-grain emission, thus favouring spinning dust [33-35]; others support the thermal hypothesis, reporting a better correlation with the total dust emission [36].

While AME is almost ubiquitous in the MW, it has been detected with high significance in three extragalactic sources only. AME has been found with pointed observations in just one star-forming region in NGC 6946 [37] and one compact radio-source in NGC 4725 ([38], even though, in this case, it does not appear to be associated with dust [39]). On the global SED, strong evidence for AME has been recently found in the Andromeda galaxy [11], with an AME component compatible with that in the MW. 


\section{A pilot K-band program at SRT}

As a preliminary project, at the beginning of 2021 we observed four galaxies at SRT in the K band. They are: NGC 3627 (M 66), NGC 4254 (M 99), NGC4736 (M 94) and NGC5055 (M63). These galaxies are part of the NIKA2 IMEGIN sample, of the DustPedia database and of the KINGFISHER project. DustPedia provides homogenised images, full UV-tosubmm global photometry and SED models [4, 10], while KINGFISHER provides radio continuum data and models at frequencies between 1 and $10 \mathrm{GHz}$ [9]. The first objective of the observations was the detection of AME, by comparing the SRT data with the available fits for the free-free and synchrotron components. Ultimately, the targets could become the first nucleus for a study of the complete UV-to-radio SED, once the NIKA2 data and the future SRT instruments cover the frequency gap between the submm and the K band (Fig. 1).

The K-band receiver was used together with the SArdinia Roach2-based Digital Architecture for Radio Astronomy back end (SARDARA; [40]). We selected two 1.2 GHz sub-bands at the edges of the K-band, at 18.6 and $24.6 \mathrm{GHz}$ (with HPBW $=57^{\prime \prime}$ and 45", respectively). These sub-bands were covered by $1.46 \mathrm{MHz}$ frequency channels in full Stokes mode. Observations were done on-the-fly over an area of $16^{\prime} \times 16^{\prime}$, centered on each galaxy, with a scan speed $2^{\prime} / \mathrm{s}$ and scan separation HPBW/3. The area was alternatively covered by scanning along orthogonal RA and Dec directions, for an efficient removal of the scan noise. Each coverage took about 20 minutes and was repeated 15 to 40 times, depending on the target, frequency and observing conditions.

The spectral cubes were reduced using the proprietary Single-dish Spectral-polarimetry Software (SCUBE; [41]) that accounts for calibration, RFIs removal, baseline removal and map-making. Maps for total and polarised intensity were made with pixel size $15^{\prime \prime}$. Total intensity maps are shown in Fig. 2. Even though some mechanisms proposed for AME are expected to produce polarised emission [25], we detected none.

\section{Preliminary results}

Using the apertures shown in Fig. 2, we measured the global flux density for our targets at 18.6 GHz and $24.6 \mathrm{GHz}$. These new datapoints were analized together with the mid-radio continuum from KINGFISHER. For NGC 4254 and NGC 4736, the SRT observations are consistent with the synchrotron and free-free fits to data for $v \leq 10 \mathrm{GHz}$. For NGC 3627 and NGC 5055, the $18.6 \mathrm{GHz}$ flux density is still compatible with the fit in [9]; the $24.6 \mathrm{GHz}$ flux density, instead, is in excess of the fit extrapolation and larger than that at $18.6 \mathrm{GHz}$ (see Fig. 1 for NGC 3627).

With the current data, it is difficult to asses if the $24.6 \mathrm{GHz}$ excess seen in NGC 3627 and NGC 5055 is due to an additional component, such as AME, or if it is just a statistical fluctuation. Observations at larger frequencies, like those that will be possible with the forthcoming Q-band receiver for SRT, are needed to confirm this finding. In fact, a model including synchrotron and free-free only can be fit to the entire $v \leq 24.6 \mathrm{Ghz}$ range, resulting in minor modifications with respect to the fit for $v \leq 10 \mathrm{GHz}$. The new parameters for the two radio components are still consistent with the spread of properties found in the KINGFISHER sample. From the free-free component, estimates of the global SFR can be obtained, which are consistent with those from the UV-to-submm SED fitting, though affected by large uncertainties [42]. Again, more precise determinations will need to wait for data at higher frequencies, or better to the full coverage of the $15 \leq v / \mathrm{GHz} \leq 300$ gap, for a simultaneous analysis of all the emission components, dust included.

Currently, we can only put upper limits on the AME contribution at $30 \mathrm{GHz}$. We find that $\operatorname{AME}(30 \mathrm{GHz}) / \mathrm{I}(100 \mu \mathrm{m})$ is at least a factor 5 smaller that the values measured in 

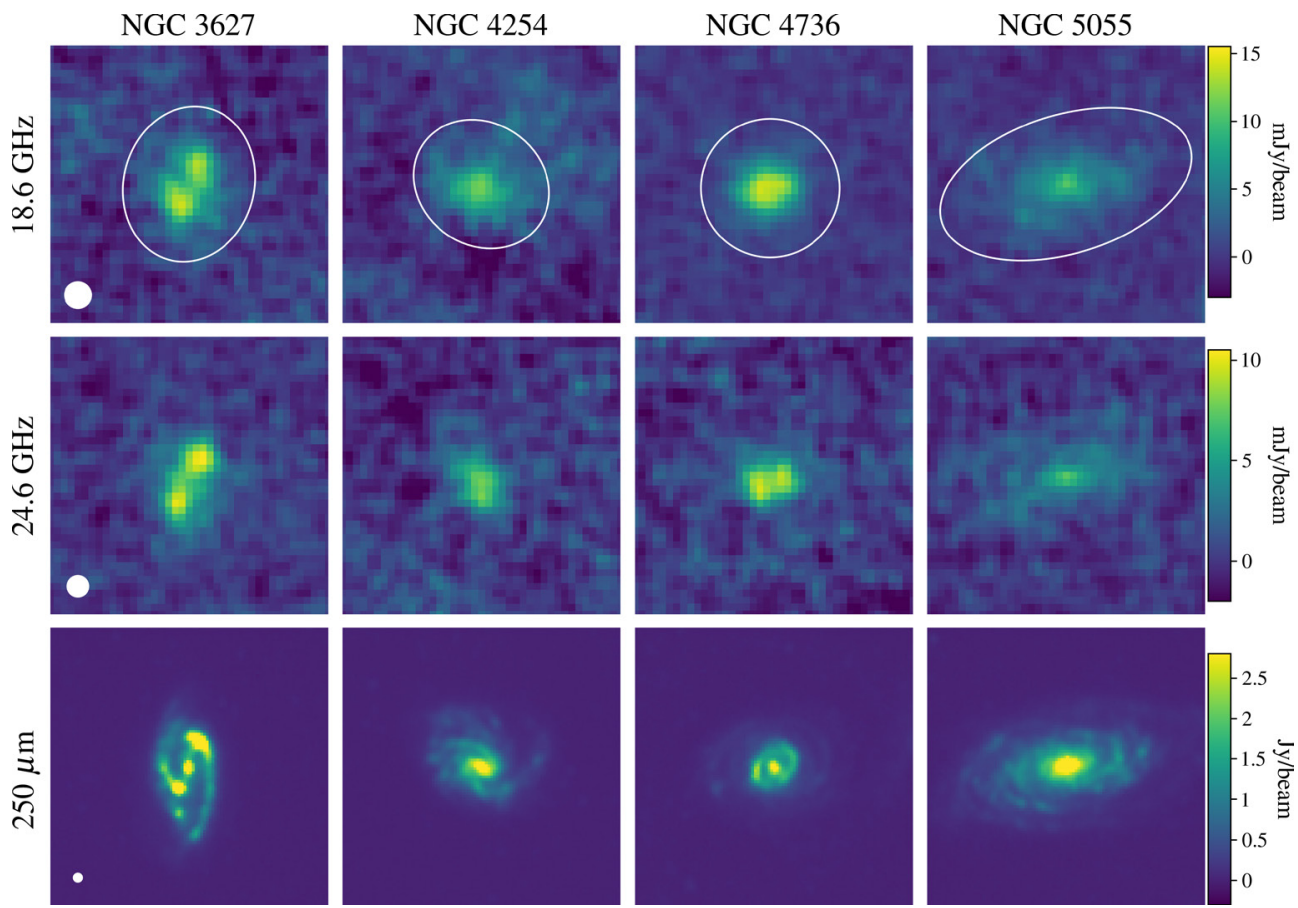

Figure 2. Total intensity maps for our targets (top and middle panels). For reference, $250 \mu \mathrm{m} \mathrm{Herschel-}$ SPIRE maps from DustPedia are shown in the bottom panels. We also show the areas used for photometry and the HPBW. Each panel is $10^{\prime} \times 10^{\prime}$.

M 31 and the MW (see the comparison between predicted AME and SRT observations in Fig. 1). This does not necessarily imply that the AME properties are different. In fact, the $\operatorname{AME}(30 \mathrm{GHz}) / \mathrm{I}(100 \mu \mathrm{m})$ ratio is an ill-defined characterisation of the AME emissivity, as it is expected to be lower in objects where the dust temperature is higher [43]. Indeed, the average dust temperatures of our galaxies, as well as those in three other targets with no AME detection [44] are higher than those of M 31 and the MW. When the AME emissivity is derived in terms of the column density of dust, available from the DustPedia analysis, the upper limits in our galaxies become consistent with the AME detections. Apparently, AME may not be detected in our targets and those in [44] because of their relatively stronger radiocontinuum than in the Milky Way and Andromeda galaxy [42]. Future searches for AME might need to consider this in the selection of targets.

\section{Acknowledgements}

We acknowledge support from the INAF mainstream 2018 program "Gas-DustPedia: A definitive view of the ISM in the Local Universe" and from grant PRIN MIUR 201720173ML3WW_001.

\section{References}

[1] Bianchi, S., De Vis, P., Viaene, S., et al., A\&A 620, A112 (2018) 
[2] Boquien, M., Burgarella, D., Roehlly, Y., et al., A\&A 622, A103 (2019)

[3] Galliano, F., MNRAS , 476, 1445 (2018)

[4] Nersesian, A., Xilouris, E. M., Bianchi, S., et al., A\&A 624, A80 (2019)

[5] Aniano, G., Draine, B. T., Hunt, L. K., et al., ApJ 889, 150 (2020)

[6] De Looze, I., Lamperti, I., Saintonge, A., et al., MNRAS 4963668 (2020)

[7] Galliano, F., Nersesian, A., Bianchi, S., et al., A\&A 649, A18 (2021)

[8] Davies, J. I., Baes, M., Bianchi, S., et al., PASP 129, 044102 (2017)

[9] Tabatabaei, F. S., Schinnerer, E., Krause, M., et al., ApJ 836, 185 (2017)

[10] Clark, C. J. R., Verstocken, S., Bianchi, S., et al., A\&A 609, A37 (2018)

[11] Battistelli, E. S., Fatigoni, S., Murgia, M., et al., ApJL 877, L31 (2019)

[12] Planck Collaboration, A\&A 594, A25 (2016)

[13] Galliano, F., Galametz, M., Jones, A. P., ARA\&A 56, 673 (2018)

[14] Compig̀ne, M., Verstraete, L., Jones, A., et al., A\&A 525, A103 (2011)

[15] Demyk, K., Meny, C., Lu, X. H., et al., A\&A 600, A123 (2017)

[16] Bianchi, S., Casasola, V., Baes, M., A\&A 631, A102 (2019)

[17] Clark, C. J. R., De Vis, P., Baes, M., et al., MNRAS 489, 5256 (2019)

[18] Katsioli, S., et al., these proceedings

[19] Ejlali, G., et al., these proceedings

[20] Murphy, E. J., Condon, J. J., Alberdi, A., et al., ASP Conference Series 517, 421 (2018)

[21] Prandoni, I., Murgia, M., Tarchi, A., et al., A\&A 608, A40 (2017)

[22] D'Alessandro, G., et al., these proceedings

[23] Orfei, A., in RadioNet Workshop on Future Trends in Radio Astronomy Instrumentation, MPIfR, Bonn (2020)

[24] Orfei, A., Carbonaro, L., Cattani, A., et al., IEEE Antennas and Propagation Magazine 52, 62 (2010)

[25] Dickinson, C., Ali-Haïmoud, Y., Barr, A., et al., New A Rev. 80, 1 (2018)

[26] Planck collaboration, A\&A 565, A103 (2014)

[27] Draine, B. T., Lazarian, A., ApJ 494, L19 (1998a)

[28] Draine, B. T., Lazarian, A., ApJ 508, 157 (1998b)

[29] Hensley, B. S., Draine, B. T., ApJ, 836, 179 (2017)

[30] Draine, B. T., Lazarian, A., ApJ 512, 740 (1999)

[31] Jones, A. P., A\&A 506, 797 (2009)

[32] Nashimoto, M., Hattori, M., Génova-Santos, R., et al., PASJ 72, 6 (2020)

[33] Ysard, N., Miville-Deschênes, M. A., Verstraete, L., A\&A 509, L1 (2010)

[34] Harper, S. E., Dickinson, C., Cleary, K., MNRAS 453, 3375 (2015)

[35] Bell, A. C., Onaka, T., Galliano, F., et al., PASJ 71, 123 (2019)

[36] Hensley, B. S., Draine, B. T., Meisner, A. M., ApJ 827, 45 (2016)

[37] Murphy, E. J., Helou, G., Condon, J. J., et al., ApJL 709, L108 (2010)

[38] Murphy, E. J., Linden, S. T., Dong, D., et al., ApJ 862, 20 (2018)

[39] Murphy, E. J., Hensley, B. S., Linden, S. T., et al., ApJL 905, L23 (2020)

[40] Melis, A., Concu, R., Trois, A., et al., Journal of Astronomical Instrumentation 7, 1850004 (2018)

[41] Murgia, M., Govoni, F., Carretti, E., et al., MNRAS 461, 3516 (2016)

[42] Bianchi, S., et al., in preparation

[43] Tibbs, C. T., Paladini, R., Dickinson, C., Advances in Astronomy 2012,124931 (2012)

[44] Peel, M. W., Dickinson, C., Davies, R. D., et al., MNRAS 416, L99 (2011) 